BULGARIAN ACADEMY OF SCIENCES

CYBERNETICS AND INFORMATION TECHNOLOGIES • Volume 15, No 4

Sofia $\bullet 2015$

Print ISSN: 1311-9702; Online ISSN: 1314-4081

DOI: $10.1515 /$ cait-2015-005

\title{
Improved Cross Entropy Measures of Single Valued Neutrosophic Sets and Interval Neutrosophic Sets and Their Multicriteria Decision Making Methods
}

\section{Jun Ye}

Department of Electrical and Information Engineering, Shaoxing University, 508 Huancheng West Road, Shaoxing, Zhejiang Province 312000, China

E-mail: yehjun@aliyun.com

\begin{abstract}
Due to some drawbacks of the cross entropy between Single Valued Neutrosophic Sets (SVNSs) in dealing with decision-making problems, the existing single valued neutrosophic cross entropy indicates an asymmetrical phenomenon or may produce an undefined (unmeaningful) phenomenon in some situations. In order to overcome these disadvantages, this paper proposes an improved cross entropy measure of SVNSs and investigates its properties, and then extends it to a cross entropy measure between interval neutrosophic sets (INSs). Furthermore, the cross entropy measures are applied to multicriteria decision making problems with single valued neutrosophic information and interval neutrosophic information. In decision making methods, through the weighted cross entropy measure between each alternative and the the ideal alternative, one can obtain the ranking order of all alternatives and the best one. The decision-making methods using the proposed cross entropy measures can efficiently deal with decision making problems with incomplete, indeterminate and inconsistent information which exist usually in real situations. Finally, two illustrative examples are provided to demonstrate the application and efficiency of the developed decision making approaches under single valued neutrosophic and interval neutrosophic environments.
\end{abstract}

Keywords: Neutrosophic set, single valued neutrosophic set, interval neutrosophic set, cross entropy; multicriteria decision making.

\section{Introduction}

Cross entropy is an important tool to judge the relation between two objects. Therefore, it can be widely applied to data analysis and classification, decision making, pattern recognition and so on. Therefore, many researchers have proposed various cross entropy measures. $\mathrm{Zadeh}[1,2]$ firstly introduced fuzzy entropy 
concept. Shannon [3] developed a cross entropy approach as information theory. $\mathrm{Ku} 1 \mathrm{lb}$ a c k and Le ible r [4] proposed a measure of the "cross entropy distance" between two probability distributions. After that, Lin [5] introduced a modified cross entropy measure. Shang and Jiang [6] presented a fuzzy cross entropy measure and a symmetric discrimination information measure between fuzzy sets. As a generalization of $\mathrm{De} \mathrm{Luca}$ and Termini [7] nonprobabilistic entropy, V l a ch o s and S e r gi a dis [8] have proposed an intuitionistic fuzzy cross entropy measure and applied it to pattern recognition, medical diagnosis and image segmentation. Then, $\mathrm{Z} \mathrm{h}$ a n g and $\mathrm{J}$ i a n g [9] defined a vague cross entropy measure by analogy with the cross entropy of probability distributions and applied it to pattern recognition and medical diagnosis and then $\mathrm{Ye}$ [10] further applied the cross entropy of vague sets to the fault diagnosis problem of turbine. Also, Y e [11] has applied the intuitionistic fuzzy cross entropy to multicriteria fuzzy decisionmaking problems. As a generalization of the vague cross-entropy [9], Ye [12] proposed an interval-valued intuitionistic fuzzy cross-entropy measure and applied it to multicriteria decision-making problems. Since a Single Valued Neutrosophic Set (SVNS) is an extension of an intuitionistic fuzzy set, Y e [13] extended the intuitionistic fuzzy cross entropy to SVNSs and proposed a single valued neutrosophic cross entropy measure, and then applied it to multicriteria decisionmaking problems with single valued neutrosophic information. However, the single valued neutrosophic cross entropy defined by $\mathrm{Y}$ e [13] has some drawbacks in some situations. For instance, it indicates an asymmetric phenomenon of the cross entropy measure of SVNSs or may produce an undefined (unmeaningful) phenomenon in some situations (details are given in the next section). In order to overcome these disadvantages, the paper proposes an improved cross entropy measure of SVNSs and investigates its properties, and then extends it to a cross entropy measure between Interval Neutrosophic Sets (INSs). Furthermore, the cross entropy measures of SVNSs and INSs are applied to multicriteria decision-making problems with single valued neutrosophic information and interval neutrosophic information.

The rest of the paper is organized as follows. Section 2 briefly describes some concepts of SVNSs, the cross entropy between SVNSs and its drawbacks in some cases. In Section 3 we propose an improved cross entropy measure between SVNSs and investigate its properties. Section 4 extends the improved cross entropy measure of SVNSs to the cross entropy measure of INSs. Section 5 applies the cross entropy measures of SVNSs and INSs to multicriteria decision making problems with single valued neutrosophic information and interval neutrosophic information. In Section 6 two illustrative examples are provided to demonstrate the application and efficiency of the developed decision making approaches under single valued neutrosophic and interval neutrosophic environments. Section 7 gives conclusions and perspectives of future work.

\section{Single valued neutrosophic cross entropy}

The neutrosophic set proposed firstly by S marandache [14] generalizes an intuitionistic fuzzy set and an interval-valued intuitionistic fuzzy set from 
philosophical point of view. It can represent uncertainty, imprecise, incomplete and inconsistent information, which the intuitionistic fuzzy set and the interval-valued intuitionistic fuzzy set cannot express. From scientific or engineering point of view, the neutrosophic set will be difficult to be applied in real science and engineering areas [15]. Therefore, as an instance of the neutrosophic set, Wang et al. [15] proposed a SVNS concept and set-theoretic operators for real scientific and engineering applications. SVNS is a generalization of an intuitionistic fuzzy set and gives us an additional possibility to represent uncertainty, imprecise, incomplete, and inconsistent information, which exists in real world, and then it would be more suitable to be applied in indeterminate information and inconsistent information measures. In the following discussion, we introduce the definition of SVNS [15].

Definition 1 [15]. Let $X$ be a space of points (objects) with generic elements in $X$ denoted by $x$. A SVNS $A$ in $X$ is characterized by a truth-membership function $T_{A}(x)$, an indeterminacy-membership function $I_{A}(x)$, and a falsity-membership function $F_{A}(x)$, where $T_{A}(x), I_{A}(x), F_{A}(x) \in[0,1]$ for each point $x$ in $X$. Therefore, a SVNS A can be expressed as

$$
A=\left\{\left\langle x, T_{A}(x), I_{A}(x), F_{A}(x)\right\rangle \mid x \in X\right\},
$$

whereas, the sums of $T_{A}(x), I_{A}(x)$ and $F_{A}(x)$ satisfy the condition

$$
0 \leq T_{A}(x)+I_{A}(x)+F_{A}(x) \leq 3 .
$$

The following inclusion, equality, complement for SVNSs $A$ and $B$ are defined, respectively, as follows [15]:

(1) $A \subseteq B$ if and only if $T_{A}(x) \leq T_{B}(x), I_{A}(x) \geq I_{B}(x), F_{A}(x) \geq F_{B}(x)$ for any $x$ in $X$,

(2) $A=B$ if and only if $A \subseteq B$ and $B \subseteq A$,

(3) $A^{c}=\left\{\left\langle x, F_{A}(x), 1-I_{A}(x), T_{A}(x)\right\rangle \mid x \in X\right\}$.

As the extension of the intuitionistic fuzzy cross entropy, Ye [13] has introduced a cross-entropy measure between SVNSs, which provides a better and more efficient mathematical framework in handling uncertain and inconsistent information.

For two SVNSs $A$ and $B$, Ye [13] defined the following single valued neutrosophic cross entropy measure between $A$ from $B$.

Definition 2 [13]. Let $A=\left\{\left\langle x_{i}, T_{A}\left(x_{i}\right), I_{A}\left(x_{i}\right), F_{A}\left(x_{i}\right)\right\rangle \mid x_{i} \in X\right\} \quad$ and $B=\left\{\left\langle x_{i}, T_{B}\left(x_{i}\right), I_{B}\left(x_{i}\right), F_{B}\left(x_{i}\right)\right\rangle \mid x_{i} \in X\right\}$ be two SVNSs $A$ and $B$ in a universe of discourse $X=\left\{x_{1}, x_{2}, \ldots, x_{n}\right\}$. Then, a single valued neutrosophic cross-entropy measure between $A$ from $B$ is defined as

$$
\text { (1) } \begin{aligned}
E(A, B) & =\sum_{i=1}^{n}\left[T_{A}\left(x_{i}\right) \log _{2} \frac{T_{A}\left(x_{i}\right)}{\left(T_{A}\left(x_{i}\right)+T_{B}\left(x_{i}\right)\right) / 2}+\left(1-T_{A}\left(x_{i}\right)\right) \log _{2} \frac{1-T_{A}\left(x_{i}\right)}{1-\left(T_{A}\left(x_{i}\right)+T_{B}\left(x_{i}\right)\right) / 2}\right]+ \\
& +\sum_{i=1}^{n}\left[I_{A}\left(x_{i}\right) \log _{2} \frac{I_{A}\left(x_{i}\right)}{\left(I_{A}\left(x_{i}\right)+I_{B}\left(x_{i}\right)\right) / 2}+\left(1-I_{A}\left(x_{i}\right)\right) \log _{2} \frac{1-I_{A}\left(x_{i}\right)}{1-\left(I_{A}\left(x_{i}\right)+I_{B}\left(x_{i}\right)\right) / 2}\right]+ \\
& +\sum_{i=1}^{n}\left[F_{A}\left(x_{i}\right) \log _{2} \frac{F_{A}\left(x_{i}\right)}{\left(F_{A}\left(x_{i}\right)+F_{B}\left(x_{i}\right)\right) / 2}+\left(1-F_{A}\left(x_{i}\right)\right) \log _{2} \frac{1-F_{A}\left(x_{i}\right)}{1-\left(F_{A}\left(x_{i}\right)+F_{B}\left(x_{i}\right)\right) / 2}\right]
\end{aligned}
$$


which also indicates the discrimination degree of $A$ from $B$. Then, it satisfies the following properties [13]:

1) $E(A, B) \geq 0$,

2) $E(A, B)=0$ if and only if $T_{A}\left(x_{i}\right)=T_{B}\left(x_{i}\right), I_{A}\left(x_{i}\right)=I_{B}\left(x_{i}\right)$, and $F_{A}\left(x_{i}\right)=F_{B}\left(x_{i}\right)$, $x_{i} \in X$,

3) $E\left(A^{c}, B^{c}\right)=E(A, B)$.

However, one can find some drawbacks of Equation (1) as follows:

1) For two SVNSs $A$ and $B$, if one of the three functions $T_{A}\left(x_{i}\right), I_{A}\left(x_{i}\right), F_{A}\left(x_{i}\right)$ is equal to 0 or one of the three functions $T_{A}\left(x_{i}\right), I_{A}\left(x_{i}\right)$ and $F_{A}\left(x_{i}\right)$ is equal to 1 for any $x_{i}$ in $X(i=1,2, \ldots, n)$, Equation (1) is undefined or unmeaningful. In this case, one cannot utilize them to calculate the cross entropy between $A$ from $B$.

2) For two SVNSs $A$ and $B$, there is $E(A, B) \neq E(B, A)$. This means that $E(A, B)$ is not symmetric.

As for the asymmetric problem of $E(A, B), \mathrm{Ye}$ [13] has modified it into a symmetric discrimination information measure for SVNSs:

$$
D(A, B)=E(A, B)+E(B, A) .
$$

The bigger the difference between $A$ and $B$ is, the larger $D(A, B)$ is.

As for the disadvantages of Equation (1), we shall propose another form of single valued neutrosophic cross entropy in the following section to overcome the disadvantages.

\section{Improved single valued neutrosophic cross entropy}

This section defines an improved cross entropy measure of SVNSs to overcome the aforementioned disadvantages in the existing single-valued neutrosophic crossentropy measure [13].

Definition 3. For any two SVNSs $A$ and $B$ in a universe of discourse $X=\left\{x_{1}, x_{2}, \ldots, x_{n}\right\}$, the cross entropy between SVNSs $A$ from $B$ is defined as follows:

$$
\begin{gathered}
N(A, B)=\sum_{i=1}^{n}\left[\sqrt{\frac{T_{A}^{2}\left(x_{i}\right)+T_{B}^{2}\left(x_{i}\right)}{2}}-\left(\frac{\sqrt{T_{A}\left(x_{i}\right)}+\sqrt{T_{B}\left(x_{i}\right)}}{2}\right)^{2}+\right. \\
+\sqrt{\frac{I_{A}^{2}\left(x_{i}\right)+I_{B}^{2}\left(x_{i}\right)}{2}}-\left(\frac{\sqrt{I_{A}\left(x_{i}\right)}+\sqrt{I_{B}\left(x_{i}\right)}}{2}\right)^{2}+ \\
+\sqrt{\frac{\left[1-I_{A}\left(x_{i}\right)\right]^{2}+\left[1-I_{B}\left(x_{i}\right)\right]^{2}}{2}}-\left(\frac{\sqrt{1-I_{A}\left(x_{i}\right)}+\sqrt{1-I_{B}\left(x_{i}\right)}}{2}\right)^{2}+ \\
\left.+\sqrt{\frac{F_{A}^{2}\left(x_{i}\right)+F_{B}^{2}\left(x_{i}\right)}{2}}-\left(\frac{\sqrt{F_{A}\left(x_{i}\right)}+\sqrt{F_{B}\left(x_{i}\right)}}{2}\right)^{2}\right] .
\end{gathered}
$$

Thus, we can derive the following Theorem 1 from the cross entropy measure of SVNSs $A$ and $B$. 
Theorem 1. For any two SVNSs $A$ and $B$ in a universe of discourse $X=\left\{x_{1}, x_{2}, \ldots, x_{n}\right\}$, the cross entropy measure $N(A, B)$ satisfies the following properties:

1) $N(A, B) \geq 0$,

2) $N(A, B)=0$ if and only if $T_{A}\left(x_{i}\right)=T_{B}\left(x_{i}\right), I_{A}\left(x_{i}\right)=I_{B}\left(x_{i}\right)$, and $F_{A}\left(x_{i}\right)=F_{B}\left(x_{i}\right)$, $x_{i} \in X$,

3) $N\left(A^{c}, B^{c}\right)=N(A, B)$,

4) $N(A, B)=N(B, A)$.

Proof:

1) Since there exists the inequality $\sqrt{\frac{x^{2}+y^{2}}{2}} \geq\left(\frac{\sqrt{x}+\sqrt{y}}{2}\right)^{2}$ for all real numbers $x$ and $y$, one can yield $N(A, B) \geq 0$.

2) The inequality $\sqrt{\frac{x^{2}+y^{2}}{2}} \geq\left(\frac{\sqrt{x}+\sqrt{y}}{2}\right)^{2}$ becomes the equality $\sqrt{\frac{x^{2}+y^{2}}{2}}=\left(\frac{\sqrt{x}+\sqrt{y}}{2}\right)^{2}$ if and only if $x=y$, thus there is $N(A, B)=0$ if and only if $A=B$, i.e., $T_{A}\left(x_{i}\right)=T_{B}\left(x_{i}\right), I_{A}\left(x_{i}\right)=I_{B}\left(x_{i}\right)$, and $F_{A}\left(x_{i}\right)=F_{B}\left(x_{i}\right), x_{i} \in X$.

3 ) It is obvious that $N\left(A^{c}, B^{c}\right)=N(A, B)$ is true.

4) It is obvious that $N(A, B)=N(B, A)$ is true.

Therefore, these proofs are completed.

It is clear that the proposed single valued neutrosophic cross entropy measure can overcome the drawbacks of the one proposed by Y e [13].

Let us consider the following example to demonstrate the efficiency of the proposed cross entropy measure.

Example 1. Let $A$ and $B$ be two SVNSs in the universe of discourse $X$, which are given by $A=\{\langle x, 1,0,0\rangle \mid x \in X\}$ and $B=\{\langle x, 0.5,0.4,0.2\rangle \mid x \in X\}$.

Obviously, Equation (1) is undefined in this case. Then, one can obtain $N(A, B)=N(B, A)=0.3736$ by using Equation (3). Thus, the proposed cross entropy can overcome the disadvantages of Equation (1). Hence, Equation (3) is more reasonable and more efficient than Equation (1).

When the differences of importance are considered in the elements in the universe, one needs to take the weight of the element $x_{i}, i=1,2, \ldots, n$, into account. In the following, we introduce a weighted cross entropy measure of SVNSs.

Let $w_{i}$ be the weight for each element $x_{i}, i=1,2, \ldots, n, w_{i} \in[0,1]$ and $\sum_{i=1}^{n} w_{i}=1$, then the weighted cross entropy measure between SVNSs $A$ from $B$ can be defined by 


$$
\begin{gathered}
N_{w}(A, B)=\sum_{i=1}^{n} w_{i}\left[\sqrt{\frac{T_{A}^{2}\left(x_{i}\right)+T_{B}^{2}\left(x_{i}\right)}{2}}-\left(\frac{\sqrt{T_{A}\left(x_{i}\right)}+\sqrt{T_{B}\left(x_{i}\right)}}{2}\right)^{2}+\right. \\
+\sqrt{\frac{I_{A}^{2}\left(x_{i}\right)+I_{B}^{2}\left(x_{i}\right)}{2}}-\left(\frac{\sqrt{I_{A}\left(x_{i}\right)}+\sqrt{I_{B}\left(x_{i}\right)}}{2}\right)^{2}+ \\
+\sqrt{\frac{\left[1-I_{A}\left(x_{i}\right)\right]^{2}+\left[1-I_{B}\left(x_{i}\right)\right]^{2}}{2}}-\left(\frac{\sqrt{1-I_{A}\left(x_{i}\right)}+\sqrt{1-I_{B}\left(x_{i}\right)}}{2}\right)^{2}+ \\
\left.+\sqrt{\frac{F_{A}^{2}\left(x_{i}\right)+F_{B}^{2}\left(x_{i}\right)}{2}}-\left(\frac{\sqrt{F_{A}\left(x_{i}\right)}+\sqrt{F_{B}\left(x_{i}\right)}}{2}\right)^{2}\right] .
\end{gathered}
$$

It is obvious that $N_{w}(A, B)$ also satisfy the four properties of Theorem 1.

Theorem 2. Let $w_{i}$ be the weight for each element $x_{i}, i=1,2, \ldots, n$, $w_{i} \in[0,1]$, and $\sum_{i=1}^{n} w_{i}=1$, then the weighted cross entropy measure $N_{w}(A, B)$ also satisfies the following properties:

1) $N_{w}(A, B) \geq 0$,

2) $N_{w}(A, B)=0$ if and only if $T_{A}\left(x_{i}\right)=T_{B}\left(x_{i}\right), I_{A}\left(x_{i}\right)=I_{B}\left(x_{i}\right)$, and $F_{A}\left(x_{i}\right)=F_{B}\left(x_{i}\right)$, $x_{i} \in X$,

3) $N_{w}\left(A^{c}, B^{c}\right)=N_{w}(A, B)$,

4) $N_{w}(A, B)=N_{w}(B, A)$.

Since the process to prove these properties is similar to that in Theorem 1, it is not repeated here.

\section{Cross entropy measure of INSs}

For real scientific and engineering applications, Wang et al. [16] have also introduced the concept of an INS, which is also a subclass of the neutrosophic set, and give the definition of an INS.

Definition 4 [16]. Let $X$ be a space of points (objects) with generic elements in $X$ denoted by $x$. An INS $A$ in $X$ is characterized by a truth-membership function $T_{A}(x)$, an indeterminacy-membership function $I_{A}(x)$, and a falsitymembership function $F_{A}(x)$, where $T_{A}(x)=\left[\inf _{A}(x), \sup T_{A}(x)\right] \subseteq[0,1]$, $I_{A}(x)=\left[\operatorname{infI}_{A}(x), \sup _{A}(x)\right] \subseteq[0,1]$, and $F_{A}(x)=\left[\inf _{A}(x), \sup F_{A}(x)\right] \subseteq[0,1]$ for each point $x$ in $X$. Then, an INS $A$ can be expressed as

$$
A=\left\{\left\langle x, T_{A}(x), I_{A}(x), F_{A}(x)\right\rangle \mid x \in X\right\}=
$$

$$
=\left\{\left\langle x,\left[\inf T_{A}(x), \sup T_{A}(x)\right],\left[\inf I_{A}(x), \sup I_{A}(x)\right],\left[\inf F_{A}(x), \sup F_{A}(x)\right]\right\rangle \mid x \in X\right\} .
$$

Obviously, the sum of $T_{A}(x), I_{A}(x)$ and $F_{A}(x)$ satisfies the condition $0 \leq \sup T_{A}(x)+\sup I_{A}(x)+\sup F_{A}(x) \leq 3$.

Especially, when the upper and lower ends of the interval values of $T_{A}(x), I_{A}(x)$ and $F_{A}(x)$ in an INS $A$ are equal, the INS $A$ reduces to SVNS $A$. However, both SVNSs and INSs are the subclasses of neutrosophic sets. 
The following expressions for INSs $A$ and $B$ are defined as follows [16]:

1) The complement $A^{c}$ for an INS $A$ is denoted as $T_{A}^{c}(x)=F_{A}(x)=$ $=\left[\inf _{A}(x), \sup F_{A}(x)\right], I_{A}{ }^{c}(x)=\left[1-\sup _{A}(x), 1-\operatorname{infI}_{A}(x)\right]$, and $F_{A}{ }^{c}(x)=T_{A}(x)=$ $=\left[\inf T_{A}(x), \sup T_{A}(x)\right]$ for any $x$ in $X$.

2) $A \subseteq B$ if and only if $\inf _{A}(x) \leq \inf T_{B}(x), \sup T_{A}(x) \leq \sup T_{B}(x)$, $\operatorname{infI}_{A}(x) \geq \operatorname{infI}_{B}(x), \operatorname{supI}_{A}(x) \geq \operatorname{supI}_{B}(x), \inf _{A}(x) \geq \operatorname{infF}_{B}(x)$, and $\sup F_{A}(x) \geq \sup F_{B}(x)$ for any $x$ in $X$.

3) $A=B$ if and only if $A \subseteq B$ and $B \subseteq A$.

Since the existing literature does not deal with the cross entropy of INSs, we can extend the improved cross entropy measure of SVNSs to the cross entropy measure of INSs.

Definition 5. For any two INSs $A$ and $B$ in a universe of discourse $X=\left\{x_{1}, x_{2}, \ldots, x_{n}\right\}$, the cross entropy between INSs $A$ from $B$ is defined as follows:

$$
\begin{aligned}
& M(A, B)=\frac{1}{2}\left\{\sum _ { i = 1 } ^ { n } \left[\sqrt{\frac{\left(\inf T_{A}\left(x_{i}\right)\right)^{2}+\left(\inf T_{B}\left(x_{i}\right)\right)^{2}}{2}}-\left(\frac{\sqrt{\inf T_{A}\left(x_{i}\right)}+\sqrt{\inf T_{B}\left(x_{i}\right)}}{2}\right)^{2}+\right.\right. \\
& +\sqrt{\frac{\left(\inf I_{A}\left(x_{i}\right)\right)^{2}+\left(\inf I_{B}\left(x_{i}\right)\right)^{2}}{2}}-\left(\frac{\sqrt{\inf I_{A}\left(x_{i}\right)}+\sqrt{\inf I_{B}\left(x_{i}\right)}}{2}\right)^{2}+ \\
& +\sqrt{\frac{\left[1-\inf I_{A}\left(x_{i}\right)\right]^{2}+\left[1-\inf I_{B}\left(x_{i}\right)\right]^{2}}{2}}-\left(\frac{\sqrt{1-\inf I_{A}\left(x_{i}\right)}+\sqrt{1-\inf I_{B}\left(x_{i}\right)}}{2}\right)^{2}+ \\
& \left.+\sqrt{\frac{\left(\inf F_{A}\left(x_{i}\right)\right)^{2}+\left(\inf F_{B}\left(x_{i}\right)\right)^{2}}{2}}-\left(\frac{\sqrt{\inf F_{A}\left(x_{i}\right)}+\sqrt{\inf F_{B}\left(x_{i}\right)}}{2}\right)^{2}\right]+ \\
& +\sum_{i=1}^{n}\left[\sqrt{\frac{\left(\sup T_{A}\left(x_{i}\right)\right)^{2}+\left(\sup T_{B}\left(x_{i}\right)\right)^{2}}{2}}-\left(\frac{\sqrt{\sup T_{A}\left(x_{i}\right)}+\sqrt{\sup T_{B}\left(x_{i}\right)}}{2}\right)^{2}+\right. \\
& +\sqrt{\frac{\left(\sup I_{A}\left(x_{i}\right)\right)^{2}+\left(\sup I_{B}\left(x_{i}\right)\right)^{2}}{2}}-\left(\frac{\sqrt{\sup I_{A}\left(x_{i}\right)}+\sqrt{\sup I_{B}\left(x_{i}\right)}}{2}\right)^{2}+ \\
& +\sqrt{\frac{\left[1-\sup I_{A}\left(x_{i}\right)\right]^{2}+\left[1-\sup I_{B}\left(x_{i}\right)\right]^{2}}{2}}-\left(\frac{\sqrt{1-\sup I_{A}\left(x_{i}\right)}+\sqrt{1-\sup I_{B}\left(x_{i}\right)}}{2}\right)^{2}+ \\
& \left.\left.+\sqrt{\frac{\left(\sup F_{A}\left(x_{i}\right)\right)^{2}+\left(\sup F_{B}\left(x_{i}\right)\right)^{2}}{2}}-\left(\frac{\sqrt{\sup F_{A}\left(x_{i}\right)}+\sqrt{\sup F_{B}\left(x_{i}\right)}}{2}\right)^{2}\right]\right\} .
\end{aligned}
$$

Similarily, $M(A, B)$ also satisfies the four properties of Theorem 1 .

Theorem 3. For any two INSs $A$ and $B$ in a universe of discourse $X=\left\{x_{1}, x_{2}, \ldots, x_{n}\right\}$, the cross entropy $M(A, B)$ satisfies the following properties:

1) $M(A, B) \geq 0$,

2) $M(A, B)=0$ if and only if $T_{A}\left(x_{i}\right)=T_{B}\left(x_{i}\right), I_{A}\left(x_{i}\right)=I_{B}\left(x_{i}\right)$, and $F_{A}\left(x_{i}\right)=F_{B}\left(x_{i}\right)$, $x_{i} \in X$,

3) $M\left(A^{c}, B^{c}\right)=M(A, B)$

4) $M(A, B)=M(B, A)$. 
Since the proof process of these properties is similar to that in Theorem 1, it is omitted here.

Taking the weight of the element $x_{i}, i=1,2, \ldots, n$, into account, we can also introduce a weighted cross entropy measure between INSs.

Let $w_{i}$ be the weight for each element $x_{i}, i=1,2, \ldots, n, w_{i} \in[0,1]$ and $\sum_{i=1}^{n} w_{i}=1$, then we have the weighted cross entropy measure between INSs $A$ and $B$ :

(6)

$$
\begin{aligned}
& M_{w}(A, B)=\frac{1}{2}\left\{\sum _ { i = 1 } ^ { n } w _ { i } \left[\sqrt{\frac{\left(\inf T_{A}\left(x_{i}\right)\right)^{2}+\left(\inf T_{B}\left(x_{i}\right)\right)^{2}}{2}}-\left(\frac{\sqrt{\inf T_{A}\left(x_{i}\right)}+\sqrt{\inf T_{B}\left(x_{i}\right)}}{2}\right)^{2}+\right.\right. \\
&+\sqrt{\frac{\left(\inf I_{A}\left(x_{i}\right)\right)^{2}+\left(\inf I_{B}\left(x_{i}\right)\right)^{2}}{2}}-\left(\frac{\left.\sqrt{\inf I_{A}\left(x_{i}\right)}+\sqrt{\inf I_{B}\left(x_{i}\right)}\right)^{2}+}{2}+\right. \\
&+ \sqrt{\frac{\left[1-\inf I_{A}\left(x_{i}\right)\right]^{2}+\left[1-\inf I_{B}\left(x_{i}\right)\right]^{2}}{2}}-\left(\frac{\sqrt{1-\inf I_{A}\left(x_{i}\right)}+\sqrt{1-\inf I_{B}\left(x_{i}\right)}}{2}\right)^{2}+ \\
&+\sqrt{\frac{\left(\inf F_{A}\left(x_{i}\right)\right)^{2}+\left(\inf F_{B}\left(x_{i}\right)\right)^{2}}{2}}-\left(\frac{\left.\sqrt{\inf F_{A}\left(x_{i}\right)}+\sqrt{\inf F_{B}\left(x_{i}\right)}\right)^{2}}{2}+\right. \\
&+ \sum_{i=1}^{n} w_{i}\left[\sqrt{\frac{\left(\sup T_{A}\left(x_{i}\right)\right)^{2}+\left(\sup T_{B}\left(x_{i}\right)\right)^{2}}{2}}-\left(\frac{\sqrt{\sup T_{A}\left(x_{i}\right)}+\sqrt{\sup T_{B}\left(x_{i}\right)}}{2}\right)^{2}+\right. \\
&+\sqrt{\frac{\left(\sup I_{A}\left(x_{i}\right)\right)^{2}+\left(\sup I_{B}\left(x_{i}\right)\right)^{2}}{2}}-\left(\frac{\sqrt{\sup I_{A}\left(x_{i}\right)}+\sqrt{\sup I_{B}\left(x_{i}\right)}}{2}\right)^{2}+ \\
&+\sqrt{\frac{\left[1-\sup I_{A}\left(x_{i}\right)\right]^{2}+\left[1-\sup I_{B}\left(x_{i}\right)\right]^{2}}{2}}-\left(\frac{\sqrt{1-\sup I_{A}\left(x_{i}\right)}+\sqrt{1-\sup I_{B}\left(x_{i}\right)}}{2}\right)^{2}+ \\
&\left.+\sqrt{\frac{\left(\sup F_{A}\left(x_{i}\right)\right)^{2}+\left(\sup F_{B}\left(x_{i}\right)\right)^{2}}{2}}-\left(\frac{\sqrt{\sup F_{A}\left(x_{i}\right)}+\sqrt{\sup F_{B}\left(x_{i}\right)}}{2}\right)^{2}\right]
\end{aligned}
$$

Especially, when $T_{A}\left(x_{i}\right)=\inf T_{A}\left(x_{i}\right)=\sup T_{A}\left(x_{i}\right), I_{A}\left(x_{i}\right)=\operatorname{infI}_{A}\left(x_{i}\right)=\sup I_{A}\left(x_{i}\right)$, and $F_{A}\left(x_{i}\right)=\operatorname{infF}_{A}\left(x_{i}\right)=\sup F_{A}\left(x_{i}\right)$ in the INS $A$ and $T_{B}\left(x_{i}\right)=\inf T_{B}\left(x_{i}\right)=\sup T_{B}\left(x_{i}\right)$, $I_{B}\left(x_{i}\right)=\operatorname{infI}_{B}\left(x_{i}\right)=\sup _{B}\left(x_{i}\right), F_{B}\left(x_{i}\right)=\inf _{B}\left(x_{i}\right)=\sup F_{B}\left(x_{i}\right)$ in the INS $B$ for any $x_{i}, i=1,2, \ldots, n$, in $X$, the INSs $A$ and $B$ reduce to the SVNSs $A$ and $B$, while (5) and (6) reduce, respectively, to (3) and (4).

It is obvious that $M_{w}(A, B)$ also satisfy the four properties of Theorem 2.

Theorem 4. For any two INSs $A$ and $B$ in a universe of discourse $X=\left\{x_{1}, x_{2}, \ldots, x_{n}\right\}$, the cross entropy $M_{w}(A, B)$ also satisfies the following properties:

1) $M_{w}(A, B) \geq 0$

2) $M_{w}(A, B)=0$ if and only if $T_{A}\left(x_{i}\right)=T_{B}\left(x_{i}\right), I_{A}\left(x_{i}\right)=I_{B}\left(x_{i}\right)$, and $F_{A}\left(x_{i}\right)=F_{B}\left(x_{i}\right)$, $x_{i} \in X$,

3) $M_{w}\left(A^{c}, B^{c}\right)=M_{w}(A, B)$,

4) $M_{w}(A, B)=M_{w}(B, A)$. 
Since the process to prove these properties is similar to that in Theorem 1, it is not repeated here.

\section{Multicriteria decision-making methods using the cross entropy measures}

A multicriteria decision making problem, where the alternatives can be evaluated according to multiple criteria, is the process of finding the best alternative among all of the feasible alternatives. In general, the multicriteria decision-making problem usually includes uncertainty, imprecise, incomplete and inconsistent information, which exists in real world. Then SVNSs and INSs can represent and handle the information. In this section multicriteria decision making methods, using the proposed cross entropy measures are developed under single valued neutrosophic and interval neutrosophic environments.

For a multicriteria decision-making problem, usually, there is a set of $m$ alternatives $A=\left\{A_{1}, A_{2}, \ldots, A_{m}\right\}$, which are to be evaluated based on a set of $n$ criteria $C=\left\{C_{1}, C_{2}, \ldots, C_{n}\right\}$. Assume that the weight of the criterion $C_{j}$, $j=1,2, \ldots, n$, entered by the decision-maker, is $w_{j}, w_{j} \in[0,1]$ and $\sum_{j=1}^{n} w_{j}=1$.

Following, two multicriteria decision-making methods using the cross entropy measures of SVNSs and INSs are developed under single valued neutrosophic and interval neutrosophic environments.

\subsection{Decision-making method using the cross entropy measure of SVNSs}

In the multicriteria decision-making problem with single valued neutrosophic information, the characteristics of an alternative $A_{i}, i=1,2, \ldots, m$, on a criterion $C_{j}$, $j=1,2, \ldots, n$, can be represented by the form of a SVNS:

$$
A_{i}=\left\{\left\langle C_{j}, T_{A_{i}}\left(C_{j}\right), I_{A_{i}}\left(C_{j}\right), F_{A_{i}}\left(C_{j}\right)\right\rangle \mid C_{j} \in C, j=1,2, \ldots, n\right\},
$$

where $T_{A_{i}}\left(C_{j}\right), I_{A_{i}}\left(C_{j}\right), F_{A_{i}}\left(C_{j}\right) \in[0,1]$ and $0 \leq T_{A_{i}}\left(C_{j}\right)+I_{A_{i}}\left(C_{j}\right)+F_{A_{i}}\left(C_{j}\right) \leq 3$ for $C_{j} \in C, j=1,2, \ldots, n$, and $i=1,2, \ldots, m$.

For convenience, the values of the three functions $T_{A_{i}}\left(C_{j}\right), I_{A_{i}}\left(C_{j}\right), F_{A_{i}}\left(C_{j}\right)$ in an SVNS $A_{i}$ are denoted by a Single Valued Neutrosophic Value (SVNV) $d_{i j}=\left\langle T_{i j}, I_{i j}, F_{i j}\right\rangle, i=1,2, \ldots, m$, and $j=1,2, \ldots, n$, which is usually derived from the evaluation of an alternative $A_{i}$ with respect to a criterion $C_{j}$ by the expert or decision maker. Thus, we can elicit a single valued neutrosophic decision matrix $D=\left(d_{i j}\right)_{m \times n}$.

For the ranking order of the alternatives in the decision-making problem, each ideal SVNV is defined as $d_{\mathrm{j}}^{*}=\langle 1,0,0\rangle, j=1,2, \ldots, n$, in the ideal alternative $A^{*}=\left\{d^{*}{ }_{1}, d^{*}{ }_{2}, \ldots, d^{*}{ }_{n}\right\}$. Then, based on Equation (4), the cross entropy between an alternative $A_{i}, i=1,2, \ldots, m$, and the ideal alternative $A^{*}$ can be expressed by 


$$
\begin{gathered}
N_{w}\left(A_{i}, A^{*}\right)=\sum_{j=1}^{n} w_{j}\left[\sqrt{\frac{T_{i j}^{2}+\left(T_{j}^{*}\right)^{2}}{2}}-\left(\frac{\sqrt{T_{i j}}+\sqrt{T_{j}^{*}}}{2}\right)^{2}+\right. \\
+\sqrt{\frac{I_{i j}^{2}+\left(I_{j}^{*}\right)^{2}}{2}}-\left(\frac{\sqrt{I_{i j}}+\sqrt{I_{j}^{*}}}{2}\right)^{2}+ \\
+\sqrt{\frac{\left[1-I_{i j}\right]^{2}+\left[1-I_{j}^{*}\right]^{2}}{2}}-\left(\frac{\sqrt{1-I_{i j}}+\sqrt{1-I_{j}^{*}}}{2}\right)^{2}+ \\
\left.+\sqrt{\frac{F_{i j}^{2}+\left(F_{j}^{*}\right)^{2}}{2}}-\left(\frac{\sqrt{F_{i j}}+\sqrt{F_{j}^{*}}}{2}\right)^{2}\right] .
\end{gathered}
$$

Then the smaller value of the cross entropy $N_{w}\left(A_{i}, A^{*}\right), i=1,2, \ldots, m$, indicates that an alternative is closer to the ideal alternative. Therefore, the ranking order of all the alternatives can be determined according to the increasing order of the cross entropy measure values. The alternative with the smallest cross entropy measure value is the best one.

\subsection{Decision-making method using the cross entropy measure of INSs}

In the multicriteria decision-making problem with interval neutrosophic information, the characteristics of an alternative $A_{i}, i=1,2, \ldots, m$, on a criterion $C_{j}$, $j=1,2, \ldots, n$, can be expressed by the following INS form:

$$
\begin{aligned}
A_{i}= & \left\{\left\langle C_{j}, T_{A_{i}}\left(C_{j}\right), I_{A_{i}}\left(C_{j}\right), F_{A_{i}}\left(C_{j}\right)\right\rangle \mid C_{j} \in C, j=1,2, \ldots, n\right\}= \\
= & \left\{\left\langleC_{j},\left[\inf T_{A_{i}}\left(C_{j}\right), \sup T_{A_{i}}\left(C_{j}\right)\right]\left[\inf I_{A_{i}}\left(C_{j}\right), \sup I_{A_{i}}\left(C_{j}\right)\right],\right.\right. \\
& {\left.\left.\left[\inf F_{A_{i}}\left(C_{j}\right), \sup F_{A_{i}}\left(C_{j}\right)\right]\right\rangle \mid C_{j} \in C, j=1,2, \ldots, n\right\}, }
\end{aligned}
$$

where $T_{A_{i}}\left(C_{j}\right), \quad I_{A_{i}}\left(C_{j}\right), \quad F_{A_{i}}\left(C_{j}\right) \subseteq[0,1]$ and $0 \leq \sup T_{A_{i}}\left(C_{j}\right)+\sup I_{A_{i}}\left(C_{j}\right)+$ $\sup F_{A_{i}}\left(C_{j}\right) \leq 3$ for $C_{j} \in C, j=1,2, \ldots, n$, and $i=1,2, \ldots, m$.

For convenience, the interval values of the three functions $T_{A_{i}}\left(C_{j}\right)=\left[\inf T_{A_{i}}\left(C_{j}\right), \sup T_{A_{i}}\left(C_{j}\right)\right], \quad I_{A_{i}}\left(C_{j}\right)=\left[\inf I_{A_{i}}\left(C_{j}\right), \sup I_{A_{i}}\left(C_{j}\right)\right]$, $F_{A_{i}}\left(C_{j}\right)=\left[\inf F_{A_{i}}\left(C_{j}\right), \sup F_{A_{i}}\left(C_{j}\right)\right]$ in an INS $A_{i}$ are denoted by an interval neutrosophic value (INV) $r_{i j}=\left\langle\left[T_{i j}^{L}, T_{i j}^{U}\right],\left[I_{i j}^{L}, I_{i j}^{U}\right],\left[F_{i j}^{L}, F_{i j}^{U}\right]\right\rangle, i=1,2, \ldots, m$, and $j=1,2, \ldots, n$, which is usually derived from the evaluation of alternative $A_{i}$ with respect to a criterion $C_{j}$ by an expert or decision maker. Thus, one can obtain an interval neutrosophic decision matrix $R=\left(r_{i j}\right)_{m \times n}$. 
In the multicriteria decision-making method, an ideal INV can be defined as $r_{j}^{*}=\left\langle\left[T_{j}^{L^{*}}, T_{j}^{U^{*}}\right]\left[\left[I_{j}^{L^{*}}, I_{j}^{U^{*}}\right]\left[F_{j}^{L^{*}}, F_{j}^{U^{*}}\right]\right\rangle=\langle[1,1],[0,0],[0,0]\rangle, j=1,2, \ldots, n\right.$, in the ideal alternative $A^{*}=\left\{r^{*}, r^{*}, \ldots, r^{*}\right\}$.

Hence, by applying Equation (6) the weighted cross entropy between an alternative $A_{i}, i=1,2, \ldots, m$, and the ideal alternative $A^{*}$ is given by the following formula:

$$
\begin{gathered}
M_{w}\left(A_{i}, A^{*}\right)=\frac{1}{2}\left\{\sum _ { j = 1 } ^ { n } w _ { j } \left[\sqrt{\frac{\left(T_{i j}^{L}\right)^{2}+\left(T_{j}^{L^{*}}\right)^{2}}{2}}-\left(\frac{\sqrt{T_{i j}^{L}}+\sqrt{T_{j}^{L^{*}}}}{2}\right)^{2}+\sqrt{\frac{\left(I_{i j}^{L}\right)^{2}+\left(I_{j}^{L^{*}}\right)^{2}}{2}}-\left(\frac{\sqrt{I_{i j}^{L}}+\sqrt{I_{j}^{L^{*}}}}{2}\right)^{2}+\right.\right. \\
\left.+\sqrt{\frac{\left[1-I_{i j}^{L}\right]^{2}+\left[1-I_{j}^{L^{*}}\right]^{2}}{2}}-\left(\frac{\sqrt{1-I_{i j}^{L}}+\sqrt{1-I_{j}^{L^{*}}}}{2}\right)^{2}+\sqrt{\frac{\left(F_{i j}^{L}\right)^{2}+\left(F_{j}^{L^{*}}\right)^{2}}{2}}-\left(\frac{\sqrt{F_{i j}^{L}}+\sqrt{F_{j}^{L^{*}}}}{2}\right)^{2}\right]+ \\
+\sum_{j=1}^{n} w_{j}\left[\sqrt{\frac{\left(T_{i j}^{U}\right)^{2}+\left(T_{j}^{U^{*}}\right)^{2}}{2}}-\left(\frac{\sqrt{T_{i j}^{U}}+\sqrt{T_{j}^{U^{*}}}}{2}\right)^{2}+\sqrt{\frac{\left(I_{i j}^{U}\right)^{2}+\left(I_{j}^{U^{*}}\right)^{2}}{2}}-\left(\frac{\sqrt{I_{i j}^{U}}+\sqrt{I_{j}^{U^{*}}}}{2}\right)^{2}+\right. \\
\left.\left.+\sqrt{\frac{\left[1-I_{i j}^{U}\right]^{2}+\left[1-I_{j}^{U^{*}}\right]^{2}}{2}}-\left(\frac{\sqrt{1-I_{i j}^{U}}+\sqrt{1-I_{j}^{U^{*}}}}{2}\right)^{2}+\sqrt{\frac{\left(F_{i j}^{U}\right)^{2}+\left(F_{j}^{U^{*}}\right)^{2}}{2}}-\left(\frac{\sqrt{F_{i j}^{U}}+\sqrt{F_{j}^{U^{*}}}}{2}\right)^{2}\right]\right\} .
\end{gathered}
$$

Thus, the smaller value of the cross entropy $M_{w}\left(A_{i}, A^{*}\right), i=1,2, \ldots, m$, indicates that an alternative is closer to the ideal alternative. Therefore, the ranking order of all the alternatives can be determined according to the increasing order of the cross entropy measure values. The alternative with the smallest cross entropy measure value is the best one.

\section{Illustrative examples}

This section provides two illustrative examples for multicriteria decision making problems to demonstrate the application and efficiency of the proposed decision making methods under single valued neutrosophic and interval neutrosophic environments.

6.1. Single valued neutrosophic decision-making problem on selecting global suppliers

Let us consider that the multicriteria decision-making problem adopted from Ye [13] is concerned with a manufacturing company, which wants to select the best global supplier according to the core competencies of suppliers. Now, suppose that there are four suppliers $\left\{A_{1}, A_{2}, A_{3}, A_{4}\right\}$ whose core competencies are evaluated by means of four criteria: (1) $C_{1}$ is the level of technology innovation; (2) $C_{2}$ is the control ability of the flow; (3) $C_{3}$ is the ability of management; (4) $C_{4}$ is the level of service. Then, the weight vector for the four criteria is $\boldsymbol{w}=(0.3,0.25,0.25,0.2)^{\mathrm{T}}$. The decision matrix of the suppliers is made up according to the four evaluating criteria. Therefore, the single valued neutrosophic decision matrix of the candidates [13] can be obtained as follows: 


$$
D=\left(\begin{array}{cccc}
\langle 0.5,0.1,0.3\rangle & \langle 0.5,0.1,0.4\rangle & \langle 0.7,0.1,0.2\rangle & \langle 0.3,0.2,0.1\rangle \\
\langle 0.4,0.2,0.3\rangle & \langle 0.3,0.2,0.4\rangle & \langle 0.9,0.0,0.1\rangle & \langle 0.5,0.3,0.2\rangle \\
\langle 0.4,0.3,0.1\rangle & \langle 0.5,0.1,0.3\rangle & \langle 0.5,0.0,0.4\rangle & \langle 0.6,0.2,0.2\rangle \\
\langle 0.6,0.1,0.2\rangle & \langle 0.2,0.2,0.5\rangle & \langle 0.4,0.3,0.2\rangle & \langle 0.7,0.2,0.1\rangle
\end{array}\right) .
$$

The proposed single valued neutrosophic decision-making method is applied to select the most desirable supplier.

By using Equation (7), one can obtain the values of the cross entropy $N_{w}\left(A_{i}, A^{*}\right), i=1,2,3,4$ :

$N_{w}\left(A_{1}, A^{*}\right)=0.2439, N_{w}\left(A_{2}, A^{*}\right)=0.2792, N_{w}\left(A_{3}, A^{*}\right)=0.2580$ and $N_{w}\left(A_{4}, A^{*}\right)=0.3034$.

According to the cross entropy measure values, the order of the four suppliers is $A_{1}, A_{3}, A_{2}$, and $A_{4}$. Hence, the best supplier is $A_{1}$.

For convenient comparison, we show the decision making results and the ones in [13] in Table 1.

Table 1. Results of single valued neutrosophic decision-making methods

\begin{tabular}{|c|c|c|}
\hline Measure/Ranking & Proposed method & Ye's method [13] \\
\hline \multirow{4}{*}{ Measure result } & $N_{\mathrm{w}}\left(A_{1}, A^{*}\right)=0.2439$ & $D\left(A_{1}, A^{*}\right)=1.1101$ \\
& $N_{\mathrm{w}}\left(A_{2}, A^{*}\right)=0.2792$ & $D\left(A_{2}, A^{*}\right)=1.1801$ \\
& $N_{\mathrm{w}}\left(A_{3}, A^{*}\right)=0.2580$ & $D\left(A_{3}, A^{*}\right)=0.9962$ \\
& $N_{\mathrm{w}}\left(A_{4}, A^{*}\right)=0.3034$ & $D\left(A_{4}, A^{*}\right)=1.2406$ \\
\hline Ranking order & $A_{1}, A_{3}, A_{2}, A_{4}$ & $A_{3}, A_{1}, A_{2}, A_{4}$ \\
\hline
\end{tabular}

From the results of Table 1, one can see that the ranking order of the four alternatives and the best choice are different from the results of Ye's method [13] and indicate the difference between $A_{1}$ and $A_{3}$. Obviously, the different measure methods may yield different results. However, since the improved cross-entropy measure in this paper can overcome the shortcomings of the existing cross-entropy measure [13], the proposed method is more reasonable and simpler than the existing method [13].

\subsection{Interval neutrosophic decision-making problem of investment alternatives}

Let us consider the multicriteria decision making problem discussed in [17]. There is an investment company, which wants to invest a sum of money in the best option. To invest the money, there is a panel with four possible alternatives: (1) $A_{1}$ is a car company; (2) $A_{2}$ is a food company; (3) $A_{3}$ is a computer company; (4) $A_{4}$ is an arms company. The investment company must take a decision according to the three criteria: (1) $C_{1}$ is the risk; (2) $C_{2}$ is the growth; (3) $C_{3}$ is the environmental impact. The weight vector of the three criteria is given by $w=(0.35,0.25,0.4)^{\mathrm{T}}$ [17].

When the four possible alternatives are to be evaluated by the expert under the above three criteria in the form of INVs, one can obtain the following interval neutrosophic decision matrix $R$ [17]:

$$
R=\left[\begin{array}{lll}
\langle[0.4,0.5],[0.2,0.3],[0.3,0.4]\rangle & \langle[0.4,0.6],[0.1,0.3],[0.2,0.4]\rangle & \langle[0.4,0.5],[0.7,0.8],[0.7,0.9]\rangle \\
\langle[0.6,0.7],[0.1,0.2],[0.2,0.3]\rangle & \langle[0.6,0.7],[0.1,0.2],[0.2,0.3]\rangle & \langle[0.8,0.9],[0.5,0.7],[0.3,0.6]\rangle \\
\langle[0.3,0.6],[0.2,0.3],[0.3,0.4]\rangle & \langle[0.5,0.6],[0.2,0.3],[0.3,0.4]\rangle & \langle[0.7,0.9],[0.6,0.8],[0.4,0.5]\rangle \\
\langle[0.7,0.8],[0.0,0.1],[0.1,0.2]\rangle & \langle[0.6,0.7],[0.1,0.2],[0.1,0.3]\rangle & \langle[0.8,0.9],[0.6,0.7],[0.6,0.7]\rangle
\end{array}\right] .
$$


Then, the developed interval neutrosophic decision-making approach is utilized to obtain the most desirable alternative(s).

By using Equation (8), one can obtain the values of the cross entropy $M_{w}\left(A_{i}, A^{*}\right), i=1,2,3,4$ :

$$
\begin{gathered}
M_{w}\left(A_{1}, A^{*}\right)=0.5872, M_{w}\left(A_{2}, A^{*}\right)=0.3643, M_{w}\left(A_{3}, A^{*}\right)=0.4895, \text { and } \\
M_{w}\left(A_{4}, A^{*}\right)=0.3724 .
\end{gathered}
$$

Thus, the ranking order of the four alternatives is $A_{2}, A_{4}, A_{3}$ and $A_{1}$. Therefore, the alternative $A_{2}$ is the best choice among the four alternatives.

For convenient comparison, we also show the decision making results and the ones in [17] in Table 2. It is worth noting that since the alternative with the largest similarity measure value in Ye's methods (1) and (2) [17] indicates the best one, the similarity measure values in [17] should be ranked in a decreasing order.

Table 2. Results of interval neutrosophic decision-making methods

\begin{tabular}{|c|c|c|c|}
\hline Measure/Ranking & Proposed method & Ye's method (1) [17] & Ye's method (2) [17] \\
\hline & $M_{w}\left(A_{1}, A^{*}\right)=0.5872$ & $S_{1}\left(A_{1}, A^{*}\right)=0.7667$ & $S_{2}\left(A_{1}, A^{*}\right)=0.7370$ \\
Measure result & $M_{w}\left(A_{2}, A^{*}\right)=0.3643$ & $S_{1}\left(A_{2}, A^{*}\right)=0.9542$ & $S_{2}\left(A_{2}, A^{*}\right)=0.9323$ \\
& $M_{w}\left(A_{3}, A^{*}\right)=0.4895$ & $S_{1}\left(A_{3}, A^{*}\right)=0.8625$ & $S_{2}\left(A_{3}, A^{*}\right)=0.8344$ \\
& $M_{w}\left(A_{4}, A^{*}\right)=0.3724$ & $S_{1}\left(A_{4}, A^{*}\right)=0.9600$ & $S_{2}\left(A_{4}, A^{*}\right)=0.9034$. \\
\hline Ranking order & $A_{2}, A_{4}, A_{3}, A_{1}$ & $A_{4}, A_{2}, A_{3}, A_{1}$ & $A_{2}, A_{4}, A_{3}, A_{1}$ \\
\hline
\end{tabular}

From the results of Table 2, one can see that the ranking order of the four alternatives and the best choice are in agreement with the results of Ye's method (2) [17] and indicate the efficiency of the proposed method. Then, the ranking orders between the proposed method and Ye's method (1) [17] and between Ye's methods [17] only indicate the difference between $A_{2}$ and $A_{4}$ in different measure methods.

For the above two examples, one can see that the multicriteria decision making methods using the cross entropy measures proposed in this paper are more suitable for real scientific and engineering applications because the proposed cross entropy measures of SVNSs and INSs cannot produce undefined phenomena.

\section{Conclusion}

To overcome the drawbacks of the cross entropy measure defined in [13], this paper has developed an improved cross entropy measure of SVNSs and investigated its properties, and then extended it to the cross entropy measure of INSs. Furthermore, the proposed cross entropy measures of SVNSs and INSs have been applied to multicriteria decision-making problems with single valued neutrosophic information and interval neutrosophic information. In the two decision-making methods, through the cross entropy measure between each alternative and the the ideal alternative, one can obtain the ranking order of all alternatives and the best one. Finally, two illustrative examples demonstrate the application and efficiency of the decision making approaches in a single valued neutrosophic setting and interval neutrosophic setting. The proposed decision making methods can efficiently deal with decision making problems with single valued neutrosophic information and interval neutrosophic information since the proposed cross entropy can overcome the drawbacks of the cross entropy defined in [13]. In future, we shall apply the 
cross entropy measures of SVNSs and INSs to other domains, such as pattern recognition, clustering analysis, and medical diagnosis.

Acknowledgment: This paper was supported by the National Natural Science Foundation of China No 71471172.

\section{References}

1. Z a d e h, L. A. Fuzzy Sets. - Information and Control, Vol. 8, 1965, pp. 338-353.

2. Z a d e h, L. A. Probability Measures of Fuzzy Events. - Journal of Mathematical Analysis and Applications, Vol. 23, 1968, pp. 421-427.

3. Sh a n n o n, C. E. A Mathematical Theory of Communication. - Bell System Technical Journal, Vol. 27, 1948, pp. 379-423.

4. K u $11 \mathrm{~b}$ a c k, S., R. A. L e i b le r. On Information and Sufficiency. - Annals of Mathematical Statistics, Vol. 4, 1951, pp. 99-111.

5. L i n, J. Divergence Measures Based on Shannon Entropy. - IEEE Transaction on Information Theory, Vol. 37, 1991, pp. 145-151.

6. S h a n g, X. G., W. S. J i a n g. A Note on Fuzzy Information Measures. - Pattern Recognition Letters, Vol. 18, 1997, pp. 425-432.

7. De Luca, A. S., S. Termini. A Definition of Nonprobabilistic Entropy in the Setting of Fuzzy Sets Theory. - Information and Control, Vol. 20, 1972, pp. 301-312.

8. V l a c h o s, I. K., G. D. S e r g i a d i s. Intuitionistic Fuzzy Information - Applications to Pattern Recognition. - Pattern Recognition Letters, Vol. 28, 2007, pp. 197-206.

9. Zhang, Q. S., S. Y. Ji ang. A Note on Information Entropy Measures for Vague Sets. Information Sciences, Vol. 178, 2008, pp. 4184-4191.

10. Y e, J. Fault Diagnosis of Turbine Based on Fuzzy Cross Entropy of Vague Sets. - Expert Systems with Applications, Vol. 36, 2009, pp. 8103-8106.

11. Y e, J. Multicriteria Fuzzy Decision-Making Method Based on the Intuitionistic Fuzzy CrossEntropy. - In: Tang, Y. C., J. Lawry, V. N. Huynh, Eds. Proc. of International Conference on Intelligent Human-Machine Systems and Cybernetics, IEEE Computer Society, Vol. 1, 2009, pp. pp. 59-61.

12. Y e, J. Fuzzy Cross Entropy of Interval-Valued Intuitionistic Fuzzy Sets and Its Optimal DecisionMaking Method Based on the Weights of Alternatives. - Expert Systems with Applications, Vol. 38, 2011, pp. 6179-6183.

13. Y e, J. Single Valued Neutrosophic Cross-Entropy for Multicriteria Decision Making Problems. Applied Mathematical Modelling, Vol. 38, 2014, pp. 1170-1175.

14. $\mathrm{S} \mathrm{m}$ a r a $\mathrm{d} \mathrm{a} \mathrm{che,} \mathrm{F.} \mathrm{A} \mathrm{Unifying} \mathrm{Field} \mathrm{in} \mathrm{Logics.} \mathrm{Neutrosophy:} \mathrm{Neutrosophic} \mathrm{Probability,} \mathrm{Set}$ and Logic. Rehoboth, American Research Press, 1999.

15. W ang, H., F. S marandache, Y. Q. Zhang, R. S underraman. Single Valued Neutrosophic Sets. - Multispace and Multistructure, Vol. 4, 2010, pp. 410-413.

16. W a n g, H., F. S m a rand a che, Y. Q. Z h ang, R. S und erra m a n. Interval Neutrosophic Sets and Logic: Theory and Applications in Computing. Hexis, Phoenix, AZ, 2005.

17. Ye, J. Similarity Measures between Interval Neutrosophic Sets and Their Applications in Multicriteria Decision Making. - Journal of Intelligent \& Fuzzy Systems, Vol. 26, 2014, pp. 165-172. 